\title{
Urbanization impact on hydrogeological regime in Jaipur urban block: A rapidly growing urban center in NW India
}

M. K. Pandit - Vinay Bhardwaj · Naveen Pareek

(C) Springer Science + Business Media, LLC 2006

The publisher regrets that this article was published in error. The article which appeared on pages 165-173 of the online issue publication has been withdrawn and will not appear in the printing of the corresponding hardcopy issue. 